\title{
ON IDEALS AND HOMOLOGY IN ADDITIVE CATEGORIES
}

\author{
LUCIAN M. IONESCU
}

Received 28 January 2001 and in revised form 26 July 2001

Ideals are used to define homological functors in additive categories. In abelian categories the ideals corresponding to the usual universal objects are principal, and the construction reduces, in a choice dependent way, to homology groups. The applications considered in this paper are: derived categories and functors.

2000 Mathematics Subject Classification: 18G50, 18A05.

1. Introduction. Categorification is by now a commonly used procedure [1, 6, 9]. The concept of an additive category generalizes that of a ring in the same way groupoids generalize the notion of groups. Additive categories were called "rings with several objects" in [14], and were studied by imitating results and proofs from noncommutative homological ring theory, to additive category theory. Alternatively, the additive category theory may be applied, as in [15], to the ring theory. Subsequent related papers adopted the "ideal theory" point of view, for example, [5], and in [17] the problem of lifting algebraic geometry to the category theory level was considered and a notion of prime spectrum of a category was defined.

In this paper, we consider the Dedekind's original aim for introducing ideals [7], and leading to the study of general rings, not only principal ideal rings (PIR). In the context of categories, we relax the requirements of an exact category for the existence of kernels and cokernels, and define homological objects in an intrinsic way, using ideals. The former are not "intrinsic" concepts. Universal constructions in category theory represent classes of morphisms in terms of a (universal) generator (a limit). The use of "coordinates" in geometry, or the use of generators and relations in algebra is opposed to the intrinsic point of view which emphasizes the "global object," which in our case is the ideal.

To fix the ideas, consider an example, kernels:

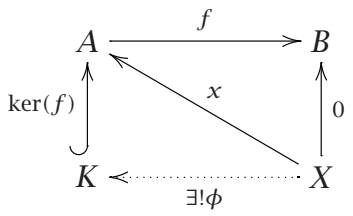

Via categorification, the morphisms whose composition with $f$ is zero is in the one object category case (ring) a right ideal. The existence of kernels in the categorical sense means that this right ideal is principal, and the universality property means that it is free as a right module, that is, the one generator forms a base, and therefore, 
it is unique modulo a "unit" (isomorphism):

$$
\operatorname{Ker}(f):=\{\operatorname{ker}(f) \circ \phi \mid \forall \phi\}=\operatorname{Ann}_{R}(f):=\{x \mid f x=0\} .
$$

Rephrasing the above equalities, $\operatorname{ker}(f)$ exists (categorical level) if and only if $\operatorname{Ann}_{R}(f)$ is a free and principal right ideal (ring theory level).

What benefit we have from this (re)interpretation? To define homology of complexes in a category, we normally assume that there exists a null family of morphisms and kernels and cokernels exist. Instead, we may consider the intrinsic approach to generalize homology in the context of additive categories, using ideals.

In Section 2, we review the notion of an ideal in a category, and introduce some new related concepts as well as operations with ideals. In Section 3, we define the notions of kernel, cokernel, image, and coimage in terms of ideals. These notions allow the definition of the homology of a complex in an additive category in an intrinsic way. This approach provides an extension of the usual methods of homological algebra, and extends the ordinary homology. If the category is exact, the homology modules are represented on projectives by the usual homology groups (see Theorem 3.10).

The applications to derived categories of abelian categories, are considered in Section 4. The theory of triangulated categories was developed to provide a setup for homological algebra in additive categories which are not exact, for example, the derived categories [3, page 45]. Our approach is the natural extension to the general case.

The construction of derived functors of functors which are not additive is considered. In Section 5, we consider axioms for an additive category, formally yielding the structure of an abelian category in terms of ideals.

2. Ideals in additive categories. Let $\mathscr{A}$ be a preadditive category, that is, a category such that for any two objects $A, B$ in $\mathscr{A}, \operatorname{Hom}(A, B)$ is an object in the category $A b$ of abelian groups. We consider the notion of ideal as originally defined by [11, page 300], and consistent with the correspondence rings-additive categories, through categorification.

Definition 2.1. An ideal (left/right) is a collection $\{I(A, B)\}_{A, B \in \mathrm{Ob}(\mathscr{A})}$ of abelian subgroups $I(A, B) \subset \operatorname{Hom}_{\mathscr{A}}(A, B)$ indexed by pairs of objects of $\mathscr{A}$, which is stable under (left/right) composition with morphisms (whenever defined).

We consider also such families of abelian subgroups indexed by objects of subcategories of $\mathscr{A}$. If $\mathscr{Y}, \mathscr{T}$ are subcategories of $\mathscr{A}$, and ideal (left/right) from $\mathscr{Y}$ to $\mathscr{T}$ is a family $\{I(A, B)\}_{A \in \mathrm{Ob}(\mathscr{S}), B \in \mathrm{Ob}(\mathcal{T})}$ of abelian subgroups $I(A, B) \subset \operatorname{Hom}_{\mathscr{A}}(A, B)$, which is stable under composition (left/right) with morphisms from $\mathscr{A}$.

An ideal (bilateral) is a left ideal which is also a right ideal. Bilateral ideals can be characterized as follows (see [13, page 36]).

Proposition 2.2. Ideals are in a one-to-one correspondence with subfunctors of Hom: $\mathscr{A}^{\mathrm{op}} \otimes \mathscr{A} \rightarrow \mathscr{A} b$.

Left (right) ideals may be viewed as families $I(A)_{A \in \mathrm{Ob}(\mathscr{A})}$ of subfunctors of the corresponding canonical representable functors $\operatorname{Hom}(A, \cdot)$. The notation $I(A)=I_{A}$ is also used. 
The condition that $I(A)$ is a subfunctor of $\operatorname{Hom}(A, \cdot)$ ensures that the family is stable under left composition with arbitrary morphisms $\phi: X \rightarrow Y$, where $A, X, Y \in \mathrm{Ob}(\mathscr{A})$ :
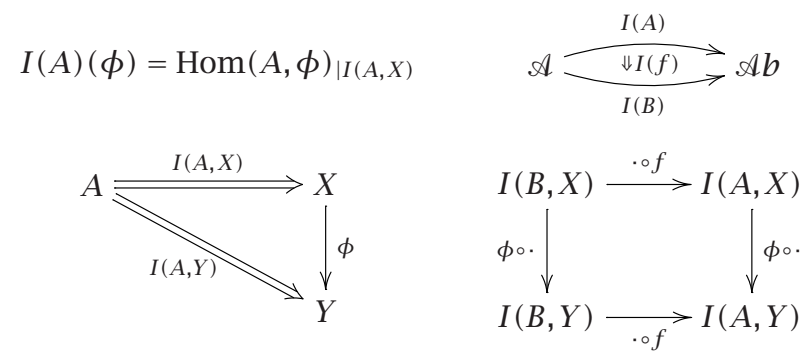

The double arrow denotes a set of morphisms, and the left diagram is 2-commutative, that is, $\eta_{A, X}$ is the 2-morphism corresponding to the inclusion of sets. Composing $\phi$ with morphisms from $I(A, X)$ may result in a proper subset of $I(A, Y)$.

The diagram from the right represents the naturality of the transformation $I(f)$ associated to a morphism $f: A \rightarrow B$. Its commutativity is equivalent to the associativity of the composition of morphisms in $\mathscr{A}$.

There is an alternative terminology for right (left) ideals. A right (left) ideal (from $A)$ to $P \in \mathrm{Ob}(\mathscr{A})$ is called a right (left) ideal in [14, page 18], and a right $P$-ideal in [15, page 140]. It is essentially a (co)sieve at $P$ [13, page 37], by adjoining null morphisms ending at $P$ if necessary (see also [2, page 171] and [17, page 139]).

The support of the left (right) ideal $I$ is defined as the full subcategory of $\mathscr{S}$ consisting of objects $A$, such that $I(A, X) \neq 0(I(X, A) \neq 0)$ for at least one object $X$ of $A$. Since any ideal can be extended trivially outside its support, we assume all ideals defined on $\mathscr{A}$.

As an example, for a given morphism $f$ consider the class of morphisms $g \circ f(f \circ g)$ which left (right) factors through $f$. It is the principal left (right) ideal generated by a morphism $f$ [17, page 142], and it is denoted by $\langle f|(|f\rangle)$, or alternatively by $\mathscr{A} f$ $(f \mathscr{A})$. As another example, consider the category $\mathcal{O}(X)$ of open sets and inclusions, canonically associated to a topological space $X$. The principal left ideal generated by an open set $U$ is essentially the family of open sets containing $U$ [13, page 70].

An ideal $I$ supported on $\mathscr{S}$ is called proper if it is different from $\mathrm{Hom}_{\mid \mathscr{S}}$ and $0_{\mid \mathscr{S}}$ (compare [17, page 145]). In particular, the left ideal supported at $A$ consisting of all morphisms with codomain the object $A$ is not a proper ideal. It is the total sieve on $A$ (maximal sieve on $A$ [13, page 38]). A maximal ideal is defined as usual as a proper ideal not contained in another proper ideal [17, page 141].

The product of two ideals (left/right) $I$ and $J$ is the ideal generated by the class of products of morphisms $f \circ g$ with $f \in I$ and $g \in J$ (zero if the class of generators is empty).

The intersection of two left (right) ideals is the class of common morphisms. As an example, if the domain of $g$ is the codomain of $f$, then the product of the left principal ideal (l.p.i.) $\langle g|$ and the r.p.i. $|f\rangle$ is a bilateral ideal equal to their intersection. It is the bilateral ideal $\langle g \circ f\rangle$ generated by their composition, and it is denoted by $\langle g \mid f\rangle$ :

$$
A \stackrel{f}{\longrightarrow} B \stackrel{g}{\longrightarrow} C, \quad\langle g \mid f\rangle=\langle g|\cap| f\rangle=\langle g|\cdot| f\rangle=\mathscr{A}(g \circ f) \mathscr{A} .
$$


If $I$ is a bilateral ideal, $\hat{A}=\mathscr{A} / I$ is the category having the same object as $\mathscr{A}$ and morphisms are cosets modulo the ideal $I$. Note that $I$ also defines a subcategory $\mathscr{I}$ by restricting the morphisms to those in $I$. If $I$ and $J \subset I$ are two ideals, then $I / J$ : $\mathscr{I} / J \otimes \mathscr{I} / J \rightarrow \mathscr{A} b$ is the quotient ideal associating to a pair of objects $(A, B)$ the quotient group $I(A, B) / J(A, B)$. To see this, apply Proposition 2.2 to the sequence of inclusions $J \subset I \subset \operatorname{Hom}_{\mathscr{A}}$, to conclude that $I / J$ is an ideal of the quotient category $\mathscr{A} / J$.

To define a quotient of left (right) ideals, we need the notion of a module [2, page 171] and [15, page 140]. A left (right) $\mathscr{A}$-module is an additive functor $M: \mathscr{A} \rightarrow \mathscr{A} b$ $\left(M: \mathscr{A}^{\text {op }} \rightarrow \mathscr{A} b\right)$. It extends in a natural way the one object case, when $\mathscr{A}$ is the one object additive category $\left\{*_{R}\right\}$ associated to a ring $R=\operatorname{End}\left(*_{R}\right)$ (tautological categorification [9]). Then an $R$-module structure $\phi: R \rightarrow \operatorname{End}(M)$ on the abelian group $M$ is the same as a functor $M: \mathscr{A} \rightarrow \mathscr{A} b$. We denote by $\mathscr{A}$-mod (mod-Ad) the category of left (right) A-modules.

DEFINITION 2.3. If $I$ and $J \subset I$ are two right ideals, then their quotient $I / J: \mathscr{A} \rightarrow$ $\bmod -\mathscr{A}$ is defined as the family of right modules $(I / J)_{A}=I_{A} / J_{A}$. Denoting for clarity $F=I_{A}, G=J_{A}$, and $\eta: F \rightarrow G$ the corresponding inclusion, we have the following diagram defining the functor $I / J$ on morphisms $(f: X \rightarrow Y)$ :

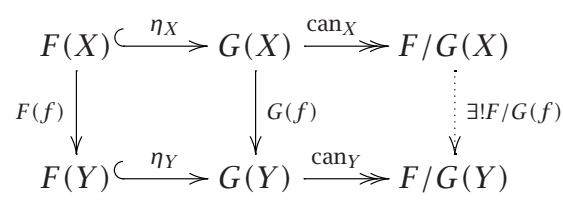

Following the noncommutative ring theory, for example, [10], we define the left (right) annihilator $\mathscr{L}(S)(\mathscr{R}(S))$ of a nonempty class of morphisms $S$ as the class of morphisms $f$ such that $f \circ a=0(a \circ f=0)$ for all $a \in S$. A left (right) ideal $I$ is called an annihilator left (right) ideal of $\mathscr{A}$ if $I=\mathscr{L}(S)(I=\mathscr{R}(S))$ for some nonempty class of morphisms $S$.

3. Homology. To define the homology of a complex in $\mathscr{A}$, we consider the families of morphisms, rather than the limits giving the usual universal objects.

Definition 3.1. The right (left) annihilator of a left (right) ideal $I$ is called the kernel ideal (cokernel ideal), and is denoted by $\operatorname{Ker}(I)$ (Coker $(I))$. The coimage ideal of a left ideal $I$ is $\operatorname{Coim}(I)=\operatorname{Coker}(\operatorname{Ker}(I))$. The image ideal of a right ideal $J$ is $\operatorname{Im}(J)=\operatorname{Ker}(\operatorname{Coker}(J))$.

If $I$ is a principal left (right) ideal generated by $f$, the following brief notation is used:

$$
\begin{aligned}
& \operatorname{Ker}(f)=\mathscr{R}(<f \mid), \quad \operatorname{Coker}(f)=\mathscr{L}(\mid f>) \\
& \operatorname{Im}(f)=\operatorname{Ker}(\operatorname{Coker}(f)), \quad \operatorname{Coim}(f)=\operatorname{Coker}(\operatorname{Ker}(f)) .
\end{aligned}
$$

From the definitions the following lemma is clear.

LEMMA 3.2. (1) $\operatorname{Ker}(I)$ and $\operatorname{Im}(I)$ are right ideals. The Coker $(I)$ and $\operatorname{Coim}(I)$ are left ideals. 
If $I=\langle f|$, then $\operatorname{Ker}(I)$ is a sieve on the domain of $f$ and $\operatorname{Im}(I)$ is a sieve on the codomain of $f$. Also Coker $(I)$ is a cosieve on the codomain of $F$ and $\operatorname{Coim}(I)$ is a cosieve on the domain of $f$.

Note that a left (right) ideal $I$ is contained in its coimage (image), since Ker and Coker reverse inclusions:

$$
I \subset J \Longrightarrow \operatorname{Ker}(J) \subset \operatorname{Ker}(I)
$$

while Im and Coim preserve inclusions.

A left (right) ideal $I$ is called closed if it coincides with its coimage (image).

LEMMA 3.3. Kernels, cokernels, images, and coimages are closed ideals, for example

$$
\operatorname{Ker}(\operatorname{Coker}(\operatorname{Ker}(I)))=\operatorname{Ker}(I), \quad \text { I left ideal. }
$$

Proof. We prove the statement corresponding to kernels. If $I$ is a left ideal, then $I \subset \operatorname{Coker}(\operatorname{Ker}(I))$, so that $\operatorname{Ker}(\operatorname{Coker}(\operatorname{Ker}(I))) \subset \operatorname{Ker}(I)$. Now, if $\psi \in \operatorname{Ker}(I)$, for all $\psi \in$ Coker $(\operatorname{ker}(I)) \psi \circ \phi=0$, and $\phi \in \operatorname{Ker}(\operatorname{Coker}(\operatorname{Ker}(I)))$. Therefore, $\operatorname{Ker}(\operatorname{Coker}(\operatorname{Ker}(I)))=$ $\operatorname{Ker}(I)$.

If $\operatorname{Ker}(f)(\operatorname{Im}(f))$ is a principal right ideal, then a generator is a kernel of $f$ in the usual sense, and is denoted by $\operatorname{ker}(f)(\operatorname{im}(f))$. Similarly for the principal left ideals $\operatorname{Coker}(f)=|\operatorname{coker}(f)\rangle$ and $\operatorname{Coim}(f)=|\operatorname{coim}(f)\rangle$.

In what follows, we use the terms kernel, cokernel, and so forth as an abbreviation for the corresponding ideals, not assumed to be principal. The following lemma is essential for defining homology in terms of ideals.

LEMMA 3.4. If the morphisms $g$ and $f$ are composable, then the following conditions are equivalent:

(a) The sequence of two morphisms is a complex: $g \circ f=0$.

(b) The image of $f$ is contained in the kernel of $g: \operatorname{Im}(f) \subset \operatorname{Ker}(g)$.

(c) The cokernel of $f$ contains the coimage of $g$ : $\operatorname{Coker}(f) \supset \operatorname{Coim}(g)$.

Proof. The sequence $g \circ f=0$ is translated as $g \in \operatorname{Coker}(f)$. Applying Ker and using Lemma 3.2, $\operatorname{Im}(f) \subset \operatorname{Ker}(g)$ follows.

Conversely, Coker reverses the previous relation

$$
\text { Coker }(\operatorname{Ker}(g)) \subset \text { Coker }(\operatorname{Ker}(\operatorname{Coker}(f))) \text {. }
$$

Since the set from left contains $g$ and the set from right equals Coker $(f)$ by Lemma 3.3, we obtain $g \in \operatorname{Coker}(f)$, that is, $g \circ f=0$.

The equivalence of (a) and (c) follows by duality.

Let $\mathscr{C} h(\mathscr{A})$ be the category of homological complexes in $\mathscr{A}$.

Definition 3.5. The right homology of the complex $C$. is the family of right modules $\mathscr{H}_{\bullet}^{R}\left(C_{\bullet}\right)$ defined as the quotient of right ideals

$$
\mathscr{H}_{n}^{R}\left(C_{\bullet}\right)=\frac{\operatorname{Ker}\left(d_{n}\right)}{\operatorname{Im}\left(d_{n+1}\right)} .
$$


The left homology of the complex $C_{\bullet}$ is the family of left modules $\mathscr{H}_{\bullet}^{L_{(}}\left(C_{\bullet}\right)$ defined as the quotient of left ideals

$$
\mathscr{H}_{n}^{L}\left(C_{\bullet}\right)=\frac{\operatorname{Coker}\left(d_{n+1}\right)}{\operatorname{Coim}\left(d_{n}\right)}
$$

Let $f_{\bullet}: C_{\bullet} \rightarrow D$. be a morphism of complexes. $\mathscr{H}_{n}^{R}\left(f_{\bullet}\right): \mathscr{H}_{n}^{R}\left(C_{\bullet}\right) \rightarrow \mathscr{H}_{n}^{R}\left(D_{\bullet}\right)$ is the natural transformation induced by $f$. on homology (see Remark 3.6 and Definition 2.3):

$$
\mathscr{H}_{n}^{R}(f)(X)([\phi])=[f \circ \phi], \quad \phi \in \operatorname{Ker}\left(d_{n}\right)(X), X \in \operatorname{Ob}(\mathscr{A}),
$$

where the brackets denote the class modulo $\operatorname{Im}\left(d_{n+1}\right)(X) . \mathscr{H}_{n}^{L}\left(f_{\bullet}\right): \mathscr{H}_{n}^{L}\left(D_{\bullet}\right) \rightarrow \mathscr{H}_{n}^{L}\left(C_{\bullet}\right)$ is the natural transformation induced by a pull-back through $f$. on homology

$$
\mathscr{H}_{n}^{L}(f)(X)([\phi])=[\phi \circ f], \quad \phi \in \operatorname{Coker}\left(d_{n+1}\right)(X), X \in \operatorname{Ob}(\mathscr{A}) .
$$

We thus obtain the functors $\mathscr{H}_{n}^{R}: \operatorname{Ch}(\mathscr{A}) \rightarrow \operatorname{Hom}(\mathscr{A}, \mathscr{A} b)$ and $\mathscr{H}_{n}^{L}: \operatorname{Ch}(\mathscr{A}) \rightarrow \operatorname{Hom}\left(\mathscr{A}^{\mathrm{op}}, \mathscr{A} b\right)$ with values $\mathscr{A}$-modules. Explicitly, the values of the right $\mathscr{A}$-module are equivalence classes of morphisms:

$$
\mathscr{H}_{n}^{R}\left(C_{\bullet}\right)(X)=\frac{\left\{\phi: X \longrightarrow C_{n} \mid d_{n} \circ \phi=0\right\}}{\left\{\phi: X \longrightarrow C_{n} \mid \operatorname{Coker}\left(d_{n-1}\right) \circ \phi=0\right\}} .
$$

The complex is exact if and only if all homology modules vanish.

REMARK 3.6. It is immediate that $\operatorname{Ker}\left(d_{n}\right)(X)=\operatorname{ker}\left(\operatorname{Hom}\left(X, d_{n}\right)\right)$, but in general $\operatorname{Im}(f)(X) \neq \operatorname{im}(\operatorname{Hom}(X, f))$ (see Example 3.9). As an immediate consequence, a chain map $f$. as above, restricts to a map between kernels.

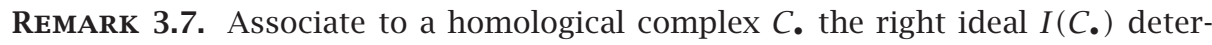
mined by the class of morphisms consisting of the morphisms of the complex together with the total sieves on the objects of the complex. Similarly, consider $J(C$.) the left ideal obtained by adjoining the cosieves supported on the complex. Then, the homology may be defined "globally" as $\operatorname{Ker}\left(J\left(C_{\bullet}\right)\right) / \operatorname{Im}\left(J\left(C_{\bullet}\right)\right)$. The direct definition is used for simplicity.

With this notion of exactness, Hom is not left exact in general. The usual sequences stating that Hom is left exact reduce to the following sequences.

Proposition 3.8. If $0 \rightarrow A \stackrel{f}{\rightarrow} B \stackrel{g}{\rightarrow} C \rightarrow 0$ is an exact sequence in the additive category $\mathcal{A}$, then for any object $X \in \mathcal{A}$, the following sequences are exact:

$$
0 \longrightarrow \operatorname{Hom}(X, A) \stackrel{f_{*}}{\longrightarrow} \operatorname{Hom}(X, B), \quad 0 \longrightarrow \operatorname{Hom}(C, X) \stackrel{g^{*}}{\longrightarrow} \operatorname{Hom}(B, X),
$$

where $f_{*}=\operatorname{Hom}(X, f)$ and $f^{*}=\operatorname{Hom}(g, X)$.

Proof. They are equivalent to $f_{*}$ and $g^{*}$ being monomorphisms $\left(\operatorname{Ker}\left(f_{*}\right)=0\right.$ and $\left.\operatorname{Ker}\left(g^{*}\right)=0\right)$. Indeed $f_{*}(\psi)=0$, that is, $f \circ \psi=0$ is equivalent to $\psi \in \operatorname{Ker}(f)$, therefore $\phi=0$. Similarly, $g^{*}(\phi)=0$, that is, $\phi \circ g=0$ is equivalent to $\phi \in \operatorname{Coker}(g)$, therefore $\phi=0$. 
EXAMPLE 3.9. A typical example showing how Hom may fail to be left exact, is the following.

Let $\mathscr{A}$ be the category of topological abelian groups and $k$ a commutative ring, for example, $k=\mathbb{Z}$. Consider the category $\mathscr{A}=k^{\mathscr{C}}$ with the same objects as $\mathscr{b}$ and with morphisms the free $k$-modules generated by the morphisms of the category $\mathscr{C}$. Then $\mathscr{A}$ is an additive category in an obvious way. The sequence $0 \rightarrow \mathbb{Q} \stackrel{j}{\rightarrow} \mathbb{R} \rightarrow 0 \rightarrow 0$ is exact in $\mathscr{A}$, since $\operatorname{Coker}(j)=0_{\mathbb{R}}$, so that $\operatorname{Im}(j)=\operatorname{Ker}\left(0_{\mathbb{R}}\right)=1_{\mathbb{R}} \mathscr{A}$. Applying $\operatorname{Hom}(\mathbb{Z}, \cdot)$ we get essentially the same sequence but in $\mathscr{A} b$, where it is not exact.

The relation to homology groups when the category is abelian is investigated next.

THEOREM 3.10. Let $C$. be a complex in the abelian category $A$. Then the right homology modules $\mathscr{H}_{n}^{R}\left(C_{\bullet}\right)$ are represented by the corresponding homology groups on the class of projective objects of $A$.

PROof. The category being abelian, the kernel ideal of $d_{n}$ and the image ideal of $d_{n+1}$ are principal and generated by the morphisms shown in the following commutative diagram:

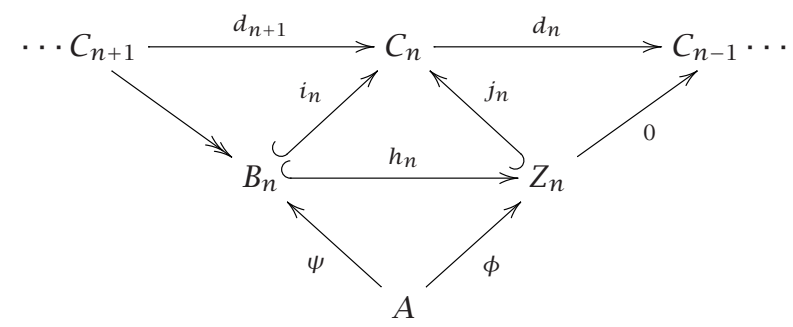

If $A$ is an arbitrary object, then $\operatorname{Ker}\left(d_{n}\right)(A)$ consists of the morphisms $j_{n} \circ \phi$ with $\phi$ arbitrary in $\operatorname{Hom}\left(A, Z_{n}\right)$, where $j_{n}=\operatorname{ker}(f)$. Similarly $\operatorname{Im}\left(d_{n+1}\right)(A)$ is parametrized by $\psi \in \operatorname{Hom}\left(A, B_{n}\right)$, where $i_{n}=\operatorname{im}\left(d_{n+1}\right)$. Applying $\operatorname{Hom}(P, \cdot)$ to the short exact sequence $0 \rightarrow B_{n} \rightarrow Z_{n} \rightarrow H_{n}\left(C_{\bullet}\right) \rightarrow 0$, with $P$ projective, we obtain the following short exact sequences:

$$
0 \longrightarrow \operatorname{Hom}\left(P, B_{n}\right) \longrightarrow \operatorname{Hom}\left(P, Z_{n}\right) \longrightarrow \operatorname{Hom}\left(P, H_{n}\left(C_{\bullet}\right)\right) \longrightarrow 0
$$

Note that $\mathscr{H}_{n}^{R}\left(C_{\bullet}\right)(P)=$ is isomorphic to $\operatorname{Hom}\left(P, Z_{n}\right) / \operatorname{Hom}\left(P, B_{n}\right)$. In this way, $\mathscr{H}_{n}^{R}\left(C_{\bullet}\right)$ is isomorphic to $\operatorname{Hom}\left(\cdot, H_{n}\left(C_{\bullet}\right)\right)$ and $H_{n}\left(C_{\bullet}\right)$ represents the right homology module $\mathscr{H}_{n}^{R}\left(C_{\bullet}\right)$, on the class of projective objects of $\mathscr{A}$.

In particular, when the category has a projective generator $U$, then the category is canonically embedded in the category of abelian groups, and the two definitions of homology functors canonically correspond.

In the following diagram, $Y$ denotes the Yoneda embedding and $h_{U}$ is the canonical representable functor which embeds the additive category $\mathscr{A}$ into the abelian category of abelian groups. Recall that a projective generator can be characterized by the 
additive functor $h_{U}$ being exact and faithful

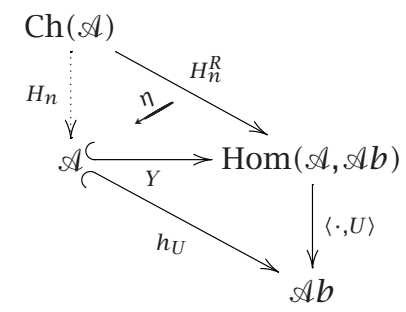

As an example, consider $\mathscr{A}=R$ - $\bmod (R$ commutative ring).

COROLLARY 3.11. If $U=R$ is the canonical projective generator of $R$-mod then there are canonical isomorphisms

$$
\mathscr{H}_{n}^{R}(\cdot)(U) \cong h_{U} \circ H_{n} \cong H_{n} .
$$

This is essentially due to the identification of the elements of an $R$-module $M$ with the morphisms $\operatorname{Hom}(R, M)$.

4. Applications. Important examples are derived categories of abelian categories, which are not necessarily abelian. We would still want to have the notions of kernel and cokernel, and so forth, in a generalized sense, and to study these categories using "abelian techniques," for example, the machinery of derived functors.

4.1. Derived categories. Recall that, given an abelian category $\mathscr{A}$, we can consider the category of complexes and chain maps $\mathrm{Ch}(\mathscr{A})$, which is also abelian.

Define the category $K(\mathscr{A})=\mathrm{Ch}(\mathscr{A}) / \mathscr{I}$ with the same objects as $\mathrm{Ch}(\mathscr{A})$ (complexes) and with morphisms homotopy classes of chain maps, that is, classes of morphisms modulo the ideal $\mathscr{I} \subset \operatorname{Hom}_{\mathrm{Ch}(\mathscr{A})}$ of null chain homotopic chain maps

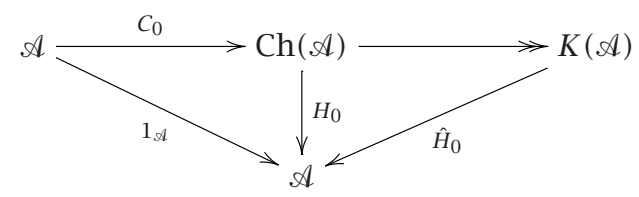

In the above diagram $C_{0}$ embeds the objects and morphisms as complexes and chain maps concentrated in degree 0 .

We do not need the actual derived category $\mathscr{D}(\mathscr{A})=K(\mathscr{A})\left[\Sigma^{-1}\right]$, which is obtained as a localization with respect to quasi-isomorphisms.

In the general case, the category $K(\mathscr{A})$, obtained by considering homotopy classes of morphisms, is no longer abelian. It may fail even to be exact, as shown by this example [3, page 45]: if $\mathscr{A}$ is a category with nontrivial extensions (e.g., abelian groups), and if $u \in \operatorname{Hom}_{\mathscr{A}}(X, Y)$ is a morphism with its image $\operatorname{Im}(u)$ being not a direct summand in $Y$, then the corresponding morphisms in $\mathrm{Ch}(\mathscr{A})$ (concentrated in degree 0 ) has no 
“classical” cokernel:

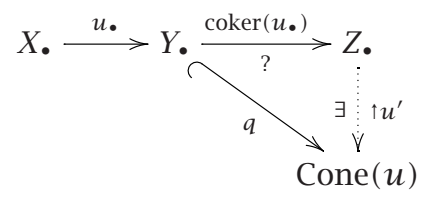

The key is that $q \circ u$ is chain homotopic to zero, and if $u$ would have a cokernel, then the short exact sequence corresponding to its image (in $\mathscr{A}$ ) must be split.

Note that the ideal $\operatorname{Coker}(u)$ always exists.

4.2. Derived functors. Since the key lemmas "lift" from generators to ideals (see [16, page 176]), homology theories can be defined for additive categories.

To illustrate that statement, we prove the homotopical lemma, leading to the machinery of derived functors.

LEMMA 4.1. Chain homotopic morphisms induce canonical isomorphims in homology

$$
f_{\bullet} \sim g_{\bullet} \Longrightarrow \mathscr{H}_{n}^{R}\left(f_{\bullet}\right)=\mathscr{H}_{n}^{R}\left(g_{\bullet}\right)
$$

Proof. If $f_{\bullet}$ and $g$. are chain homotopic morphisms with a chain homotopy $s_{\bullet}$, then

$$
\mathscr{H}_{n}^{R}\left(f_{n}-g_{n}\right)([\phi])=\left[\left(d_{n+1}^{\prime} s_{n}+s_{n-1} d_{n}\right) \circ \phi\right]=0,
$$

since $\phi \in \operatorname{Ker}\left(d_{n}\right)$ and $d_{n+1}^{\prime} \circ h \in \operatorname{Im}\left(d_{n+1}^{\prime}\right)$ for any composable morphims $h$ in $\mathscr{A}$.

There is an analog of the connecting morphism [8, page 99]. From the start we point out that additional requirements on the category are necessary to enable the familiar long exact sequence theorem from homology. The relation with the requirement for distinguished triangles [3], will be investigated elsewhere. The additional requirement is the analog of the usual axiom for abelian categories, that is, "monomorphisms are kernels" (see Section 5).

DEFINITION 4.2. An additive category, such that principal right ideals generated by monomorphisms and principal left ideals generated by epimorphisms are closed ideals (see Lemma 3.3)

$$
\mu A=\operatorname{Im}(\mu), \quad \mu \text { mono, } \quad A \eta=\operatorname{Coim}(\eta), \quad \eta \text { epi, }
$$

will be called regular.

In such categories, due to the mechanism of long exact sequences (see [8, page 121]), derived functors form a connected sequence of functors [4, 12].

THEOREM 4.3. In a regular additive category, restricting to projective objects and morphisms, there are natural transformations $\delta_{n}^{R}$, such that $\left(\mathscr{H}_{n}^{R}, \delta_{n}^{R}\right)$ is a connected sequence of functors. 
Proof. Given a short exact sequence of complexes in $\mathscr{A}$

$$
0 \longrightarrow A_{\bullet} \stackrel{f}{\longrightarrow} B . \stackrel{g}{\longrightarrow} C \cdot \longrightarrow 0,
$$

we prove the existence of the connecting transformation $\omega: \operatorname{Ker}\left(d_{n+1}^{C}\right) \rightarrow \mathscr{H}_{n}^{R}\left(A_{\bullet}\right)$, defined on projectives of $\mathscr{A}$. The rest of the proof follows the standard one, where "elements," for example, $c \in C$, are replaced by generalized elements $c: P \rightarrow C$.

Let $P$ be a projective object in $\mathscr{A}$, and let $c: P \rightarrow C$ be a morphism belonging to $\operatorname{Ker}\left(d_{n+1}^{C}\right)(P)$

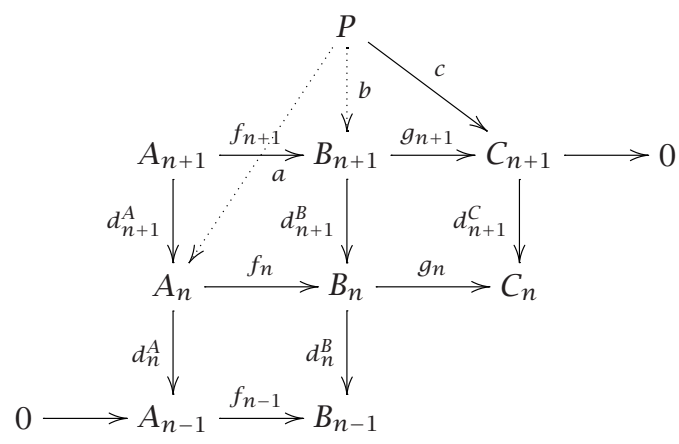

Adapting the proof from [8, page 99], there is a lift of $c$ to $B$, denoted $b$. Then $d_{n+1}^{B} \circ b \in$ $\operatorname{Ker}\left(g_{n}\right)=\operatorname{Im}\left(f_{n}\right)$. The additional assumption ensures that this morphism factors through $f_{n}$, yielding a map $a \in \operatorname{Ker}\left(d_{n}^{A}\right)(P)$. Indeed, $f_{n-1} \circ d_{n}^{A} \circ a=0$ since $B$. is a complex, and $\operatorname{Ker}\left(f_{n-1}\right)=\operatorname{Im}(0)=0$, implies $d_{n}^{A} \circ a=0$.

We may check as usual that the map $\omega(c)=a+\operatorname{Im}\left(d_{n}^{A}\right)$, valued in $\mathscr{H}_{n}^{R}\left(A_{\bullet}\right)(P)$, is independent of the choice of $b$, and it vanishes on $\operatorname{Im}\left(d_{n+2}^{C}\right)$. It thus defines the required morphism $\delta_{n}^{R}(P)$.

REMARK 4.4. There is no analog of [8, Lemma 5.1, page 99], since Ker and Coker have different "chirality." However there is a pairing

$$
\tilde{\delta}: \operatorname{Ker}\left(d_{n}^{C}\right) \otimes \operatorname{Coker}\left(d_{n+1}^{A}\right) \longrightarrow \operatorname{Hom}_{\mathscr{A}},
$$

defined with the above notations by $\tilde{\delta}_{(P, Q)}(c, d)=d \circ a$, where $d \in \operatorname{Hom}_{\mathscr{A}}\left(A_{n}, Q\right)$.

This approach allows us to generalize the construction of derived functors to the case of nonabelian functors.

More precisely, for any category we may consider the category with the same objects, and with $\operatorname{Hom}(X, Y)$ the free module generated by the corresponding morphisms of the original category. Enriching the category in this way ("linearization") may be viewed as the categorification of the group ring construction. In this way, we may apply the machinery of derived functors in the general case of an arbitrary category.

5. Additive categories and abelian category axioms. We briefly consider the abelian category axioms [16, page 83 ] in terms of ideals. 
The goal is to find the additional axioms on an additive category, providing the same flexibility as abelian categories. The author did not find an ideal-theoretic reformulation of the axioms of an abelian category, although Definition 4.2 points in this direction.

Recall that we use the term kernel as an abbreviation for the kernel ideal. The analog of the axioms $(K)$ and $\left(K^{\circ}\right)$ of an abelian category [16, page 83], hold by definition.

LEMMA 5.1. Let $A$ be an additive category. Then

$\left({ }^{\prime} K\right)$ Every morphism $f$ has a kernel $\operatorname{Ker}(f)$;

$\left({ }^{\circ} K^{\circ}\right.$ Every morphism $f$ has a cokernel Coker $(f)$;

(1) $f$ is a monomorphism if and only if $\operatorname{Ker}(f)=0$;

(2) $f$ is an epimorphism if and only if $\operatorname{Coker}(f)=0$.

Proof. The statements are immediate consequences of the definitions.

The analog of the axioms $(N)$ and $\left(N^{\circ}\right)[16$, page 83] are

$\left({ }^{\prime} N\right)$ For every monomorphism $f$, the right ideal $|f\rangle$ is a kernel ideal $(|f\rangle=\operatorname{Ker}(I)$ for some left ideal $I$ ).

$\left({ }^{\circ} N^{\circ}\right)$ For every epimorphism $f$, the left ideal $\langle f|$ is a cokernel ideal $(\langle f|=\operatorname{Coker}(I)$ for some right ideal $I$ ).

Note that in an abelian category the above statements reduce to the usual axioms.

LEMMA 5.2. If $A$ is an abelian category then $(N)$ and $\left({ }^{\prime} N\right)$ are equivalent. Dually, $\left(N^{\circ}\right)$ and $\left({ }^{\prime} N^{\circ}\right)$ are equivalent.

PRoof. If $f \mathscr{A}$ is a kernel ideal $\operatorname{Ker}(I)$, and therefore closed, $f \mathscr{A}=\operatorname{Ker}(J)$ with $J=$ Coker $(\operatorname{Ker}(I))$ the closure of $I$. Then $J=\operatorname{Coker}(f \mathscr{A})$ is a principal left ideal generated by $h=\operatorname{coker}(f)$ and $f \mathscr{A}=\operatorname{Ker}(\mathscr{A} h)=\operatorname{Ker}(h)=\operatorname{ker}(h) \mathscr{A}$. Now it is easy to see that two monomorphisms, $f$ and $g=\operatorname{ker}(h)$, generate the same right ideal if and only if they have isomorphic domains $f=g \circ s$ ( $s$ isomorphism). Therefore $f$ is the kernel (in the usual sense) of a morphism.

REMARK 5.3. From [10, page 2], the axiom $\left({ }^{\prime} N\right)$ (dually $\left({ }^{\circ} N^{\circ}\right)$ ) can be rephrased as "any right regular element $f$ (no right zero-divisors/monomorphism) generates an annihilator right ideal $f \mathscr{A}$." It further demonstrates the similarity between the ring theory and the additive category theory, as the "ring with several objects" case.

Proposition 5.4. The axiom (' $N$ ) holds if and only if any monomorphism $f$ generates its image ideal: $|f\rangle=\operatorname{Im}(f)$.

$\left({ }^{\circ} N^{\circ}\right)$ holds if and only if any epimorphism $g$ generates its coimage ideal: $\langle g|=$ $\operatorname{Coim}(g)$.

Proof. We only prove the first statement, since the second statement follows by duality. If $|f\rangle=f \mathscr{A}$ is a kernel ideal $\operatorname{Ker}(I)$, then it is closed and $\operatorname{Im}(f)=$ $\operatorname{Ker}(\operatorname{Coker}(f \mathscr{A}))$ equals $f \mathscr{A}$.

For the converse, let $f$ be a monomorphism, and assume $f \mathscr{A}=\operatorname{Im}(f)$. Then $f \mathscr{A}=$ $\operatorname{Ker}(I)$ with $I=\operatorname{Coker}(f)$.

COROLlary 5.5. A regular additive category satisfies the ideal-theoretic axioms $(' K),\left({ }^{\circ} K^{\circ}\right),\left({ }^{\prime} N\right)$, and $\left({ }^{\prime} N^{\circ}\right)$. 
It is not clear what the analog for the epi-mono factorization axiom (AB2) [15, page 84] should be, and allowing the usual epi-mono based type of arguments. We only note that the rows in the next diagram are exact, that is, the kernel of the left ideal based at $A$ equals the image of the right ideal based at $A$

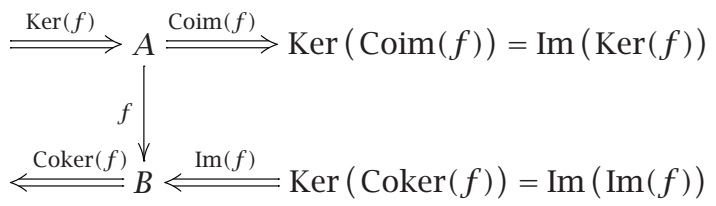

and that in general $f$ is common to both $\operatorname{Im}(f)$ and Coim $(f)$.

6. Conclusions and further developments. The aim of "categorifying ideal theory" is manifold. First, to avoid the familiar "unique-up-to-a-unit" of arithmetic in principal ideal rings and to define an "intrinsic" homology in additive categories. Second, with ideals replacing generators, a greater degree of generality is achieved. In additive categories, the analog of two basic axioms in abelian categories are consequences of the definitions.

In this way, it is possible to develop the usual formalism of homological algebra in derived categories, based on short exact sequences. Then, a natural problem is to determine the relationship between this approach and the established formalism of distinguished triangles.

ACKNOWLEDGMENTS. I thank the referees for recommendations, especially for helping the author to avoid a false claim (Theorem 4.3).

\section{REFERENCES}

[1] J. C. Baez and J. Dolan, Categorification, Higher Category Theory (Evanston, IL, 1997), Contemp. Math., vol. 230, American Mathematical Society, Rhode Island, 1998, pp. 1-36.

[2] K. Baumgartner, Structure of additive categories, Cahiers Topologie Géom. Différentielle 16 (1975), no. 2, 169-213.

[3] A. Borel, P.-P. Grivel, B. Kaup, A. Haefliger, B. Malgrange, and F. Ehlers, Algebraic DModules, Perspectives in Mathematics, vol. 2, Academic Press, Massachusetts, 1987.

[4] H. Cartan and S. Eilenberg, Homological Algebra, Princeton University Press, New Jersey, 1956.

[5] J. D. Christensen, Ideals in triangulated categories: phantoms, ghosts and skeleta, Adv. Math. 136 (1998), no. 2, 284-339, http://arxiv.org/abs/math.AT/9807071.

[6] L. Crane and D. N. Yetter, Examples of categorification, Cahiers Topologie Géom. Différentielle Catég. 39 (1998), no. 1, 3-25.

[7] H. M. Edwards, Mathematical ideas, ideals, and ideology, Math. Intelligencer 14 (1992), no. 2, 6-19.

[8] P. J. Hilton and U. Stammbach, A Course in Homological Algebra, 2nd ed., Graduate Texts in Mathematics, vol. 4, Springer-Verlag, New York, 1997.

[9] L. M. Ionescu, On categorification, http://arxiv.org/abs/math.CT/9906038, 1999.

[10] A. V. Jategaonkar, Left Principal Ideal Rings, Lecture Notes in Mathematics, vol. 123, Springer-Verlag, Berlin, 1970.

[11] G. M. Kelly, On the radical of a category, J. Austral. Math. Soc. 4 (1964), 299-307. 
[12] S. Mac Lane, Homology, Die Grundlehren der mathematischen Wissenschaften, vol. 114, Academic Press, New York, 1963.

[13] S. Mac Lane and I. Moerdijk, Sheaves in Geometry and Logic: A First Introduction to Topos Theory, Universitext, Springer-Verlag, New York, 1992.

[14] B. Mitchell, Rings with several objects, Advances in Math. 8 (1972), 1-161.

[15] R. Street, Ideals, radicals, and structure of additive categories, Appl. Categ. Structures 3 (1995), no. 2, 139-149.

[16] J. R. Strooker, Introduction to Categories, Homological Algebra and Sheaf Cohomology, Cambridge University Press, Cambridge, 1978.

[17] S.-H. Sun and A. Dhillon, Prime spectra of additive categories. I, J. Pure Appl. Algebra 122 (1997), no. 1-2, 135-157.

Lucian M. IONescu: Department of Mathematics, IlLinois State University, NoRmal, IL 61790-4520, USA

E-mail address: 1miones@i1stu.edu 


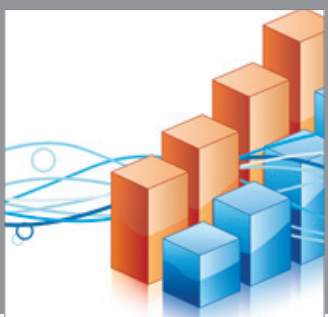

Advances in

Operations Research

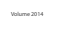

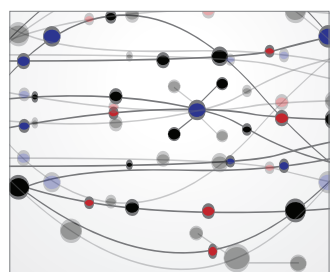

\section{The Scientific} World Journal
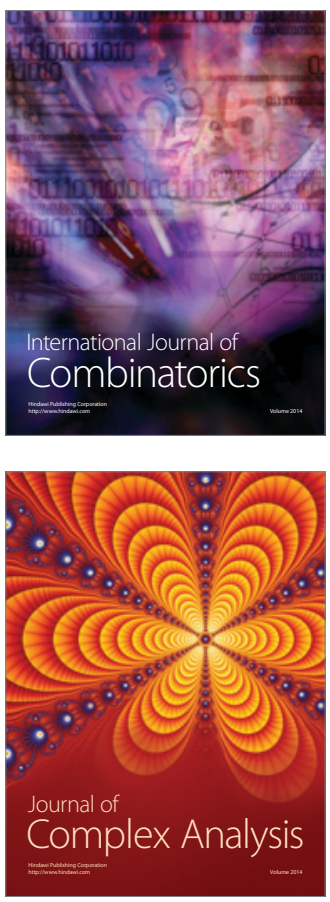

International Journal of

Mathematics and

Mathematical

Sciences
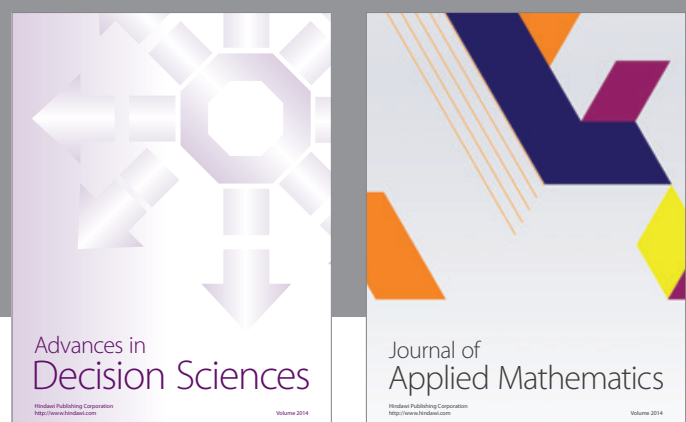

Journal of

Applied Mathematics
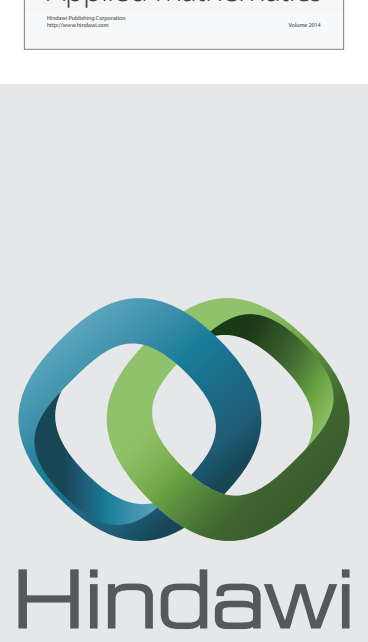

Submit your manuscripts at http://www.hindawi.com
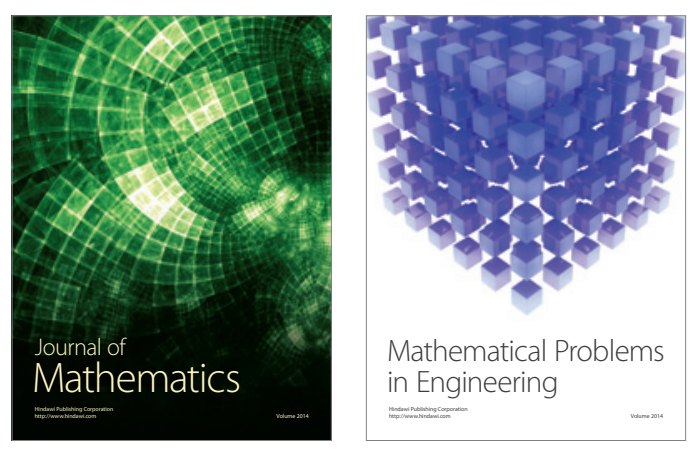

Mathematical Problems in Engineering
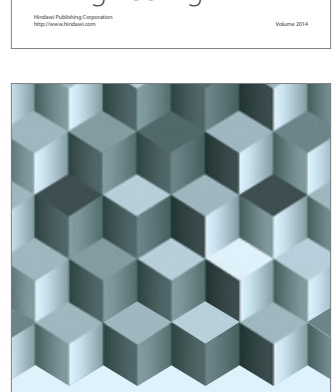

Journal of

Function Spaces
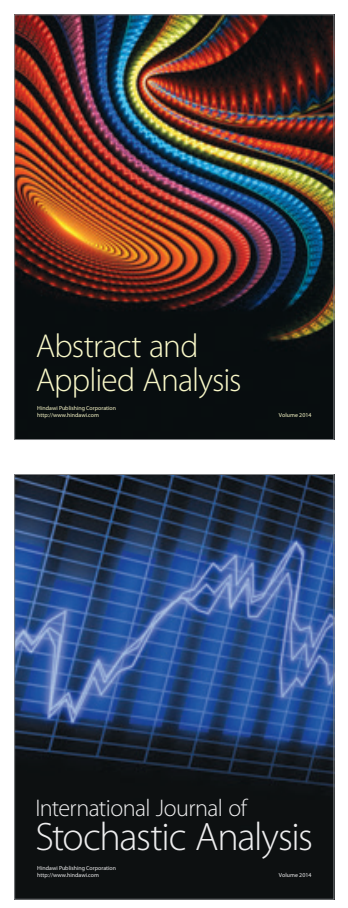

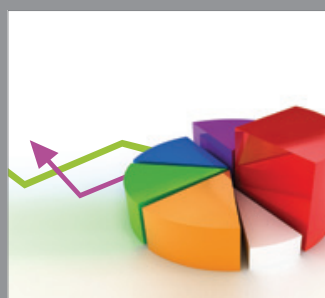

ournal of

Probability and Statistics

Promensencen
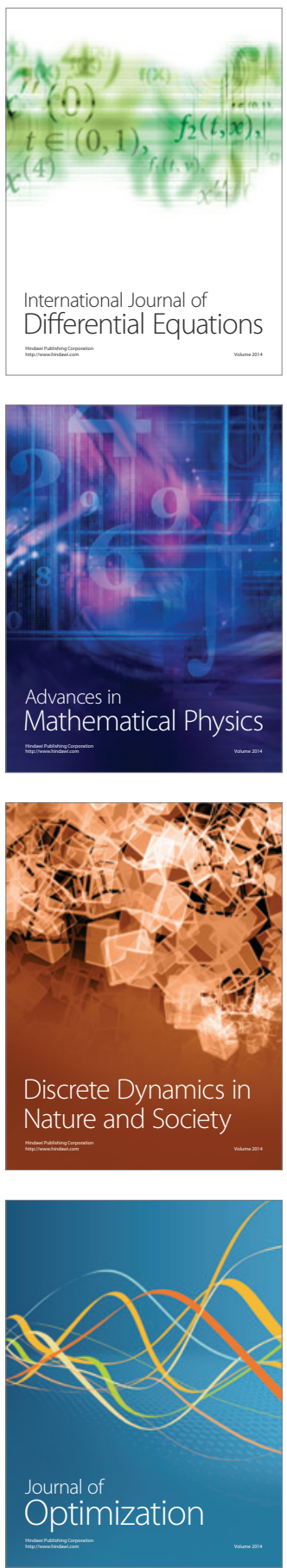